\title{
Interventions Using High-Technology Communication Devices: A State of the Art Review
}

\author{
Susan Baxter ${ }^{\mathrm{a}}$ Pam Enderby $^{\mathrm{a}}$ Philippa Evans $^{\mathrm{a}}$ Simon Judge ${ }^{\mathrm{b}}$ \\ aSchool of Health and Related Research, University of Sheffield, Sheffield, and bBarnsley District General Hospital \\ Foundation Trust, Barnsley, UK
}

\section{Key Words}

Augmentative and alternative communication - Systematic review - Assistive technology

\begin{abstract}
Background/Aims: In the last 20 years the range of hightechnology augmentative and alternative communication (AAC) aids has rapidly expanded. This review aimed to provide a 'state of the art' synthesis, to provide evidence-based information for researchers, potential users and service providers. Methods: Electronic databases were searched from 2000 to 2010, together with reference lists of included papers and review papers. The review considered work of any design which reported an intervention using high-tech AAC with people who have communication difficulties (excluding those with solely hearing or visual loss) published in peer-reviewed journals. Results: Sixty-five papers reporting interventions using high-tech AAC were identified. There was evidence that high-technology AAC may be beneficial across a range of diagnoses and ages. The evidence, however, is currently drawn from studies using designs considered to be at high risk of bias. Conclusion: The review suggests that the high level of individual variation in outcome
\end{abstract}

requires a greater understanding of characteristics of clients who may or may not benefit from this technology. Also, the wide range of outcomes measured requires further work in the field to establish what a 'good outcome' from intervention may be.

Copyright $\odot 2012$ S. Karger AG, Basel

\section{Introduction}

Augmentative and alternative communication (AAC) systems have great potential to enhance the lives of individuals with communication difficulties by promoting interlinked elements of independence, social relationships and education. The American Speech-Language Hearing Association [1] defines AAC as a set of procedures and processes for maximising functional and effective communication. The term encompasses unaided modes that rely on a user's body to convey messages, for example gestures, signs and facial expressions, and aided AAC requiring a transmission device. These devices may be electronic and commonly referred to as high-technology AAC, or non-electronic and described as low-technology assistive devices [1].

\section{KARGER}

Fax +4161306 1234 E-Mail karger@karger.ch www.karger.com
(C) 2012 S. Karger AG, Basel

1021-7762/12/0643-0137\$38.00/0

Accessible online at:

www.karger.com/fpl
Susan Baxter

Section of Public Health

School of Health and Related Research, Regent Court

30 Regent Street, Sheffield S1 4DA (UK)

Tel. +44 114222 0852, E-Mail s.k.baxter@ sheffield.ac.uk 
While there is some lack of clarity in the field regarding terminology, low-technology (low-tech) systems or devices are usually considered to include communication books or boards (non-powered), written words, photographs, line drawings and pictograms. High-technology (high-tech) systems are commonly distinguished by being powered. The literature describes custom-made communication aids which provide voice output (VOCAs), also referred to as speech-generating devices (SGDs). In addition, there is software which can be used on standard personal computers or laptops which provide a voice output. Technology is also available which enables people to access a personal computer or laptop to achieve written communication output.

Authors have described how practitioners face challenges in successfully using these devices with clients. Campbell et al. [2] for example highlighted that practitioners are unsure of when and how to implement AAC systems due to a paucity of research evidence. Schlosser [3] described how practitioners faced a difficult task when matching appropriate systems to individuals with disabilities. It has also been suggested [4] that practitioners and users may have limited access to available systems or services due to funding issues and limited specialist knowledge.

If speech-language pathologists are to include this technology in therapy interventions, there is a requirement for research evidence to be readily accessible to support evidence-based practice regarding which devices should be provided for who and at which point in treatment. High-tech AAC systems are expensive to purchase and repair, and funding may fail to meet total device costs, or include adequate speech-language pathology support [5]. The training of communication partners is also a neglected area for funding.

Decision-making regarding costly provision requires effectiveness evidence to draw upon. While there is a growing body of research exploring the use of high-tech aids, published reviews to date have tended to consider predominantly low-tech aids. Systematic reviews which have included both high- and low-tech aids have tended to examine use in specific clinical populations $[2,6]$. Authors of these reviews have suggested that much available evidence regarding AAC is inconclusive, in particular in regard to generalisation and maintenance of use. Iacono et al. [5] reported that there has been a tendency to provide high-tech aids when low-tech aids may offer advantages. In addition, the definition of outcomes to be measured offers a considerable challenge when the goal of intervention encompasses enabling individuals to access life [7].
In view of the increasing range of sophisticated technology being developed to support people with communication impairments, it is important to examine published work regarding the interventions using these devices. Evaluation of study findings is needed in order to provide evidence-based information for funders, potential users, and service managers, and to underpin evidence-based practice amongst speech-language pathologists [8]. This review therefore was undertaken as a 'state of the art' review [9] to present an assessment of the current state of knowledge in the field. The work encompassed both quantitative intervention studies and qualitative papers reporting views of service users and providers. Findings regarding the qualitative studies are reported elsewhere [10]. In this paper we consider primary studies reporting evaluations of interventions.

\section{Methods}

Search Strategy

Relevant published literature was identified via searching of the CINAHL, Cochrane Library, EMBASE, MEDLINE, PsychINFO, CSA, and Web of Science electronic databases. Search terms used related to conditions (for example learning disability, cerebral palsy), impairment terms (such as language disorder, communication impairment), AAC terms (such as speech-generating device, assistive aids) and finally commonly used devices (such as Toucan $^{\mathrm{TM}}$ ). The full search strategy may be obtained from the authors. In addition to this electronic database searching, we scrutinised the reference list of included papers and review papers for additional citations of relevance.

\section{Inclusion/Exclusion Criteria}

The review considered studies of any design published in peerreviewed journals between 2000 and 2010 that were reported in English. The population under consideration was any person who had a communication difficulty not resulting from a primary auditory or visual loss. The review also encompassed studies reporting data from relatives/significant others of these people with communication difficulties, together with staff delivering services. The review examined 'high-technology' communication devices only. Due to some inconsistency of terminology in the field we took the pragmatic decision to define high-tech devices by exclusion as those alternative and augmentative communication methods or devices which are powered and cannot be described as low technology. Software that could be used only as a treatment tool during therapy sessions was excluded, for example, computer programs used for word finding drills or articulation practice.

\section{Quality Appraisal}

Due to the inclusion criteria encompassing work across the hierarchy of study designs we assessed the quality of the included papers using the quality domains developed by West et al. [11]. This framework identifies five quality domains to be considered when appraising the risk of bias in studies encompassing: the 
comparability of participants; the intervention; outcome measurement; analysis method, and funding source. Study design terminology is used variously by different researchers in different disciplines. We adopted the typology used by the Centre for Reviews and Dissemination [12], which classifies experimental studies as those which allocate participants to intervention or control groups (randomised controlled trials or quasi-experimental studies), and observational designs where interventions are determined by usual practice or 'real-world' choices rather than allocation. These include cohort studies which compare participants who did or did not receive an intervention over time, case-control studies which match 'cases' and 'controls' from the same population, before and after studies where measurements are taken at baseline and follow-up, case series designs where there may be a single measurement point or observations taken over time, and case studies with a single participant.

\section{Selection of Publications for Review}

The initial search retrieved 2,883 unique citations. All retrieved literature was screened at title and abstract level for relevance, and those that had potential for inclusion were taken through to full paper appraisal and extraction of data. Following the initial sifting 299 papers were examined as full papers with inclusions and exclusions checked by a second member of the research team. A flow chart illustrating the process of inclusion and exclusion is available from the authors.

\section{Results}

\section{Study Characteristics}

The review identified 65 papers, most with small numbers $(n=1-5)$ of participants. The studies encompassed work from ten different countries (33 North America, 7 Italy, 6 Germany, 5 Australia, 5 UK, 4 Sweden, 1 each from South Africa, Japan, New Zealand, and The Netherlands). The country of origin of one paper was unclear. Interventions using the full range of high-tech devices encompassing VOCA/SGDs, voice output computer software, and input/controls such as speech recognition technology and brain-computer interfaces were reported. An extraction table detailing each study is available from the authors.

\section{Study Quality}

Using the appraisal outlined above, the most significant quality issues noted across the set were: a preponderance of case study and case series designs; small sample size (only three papers reported work with 10 or more participants); an absence of studies using comparator interventions, and no work using random allocation. In many cases the intervention was ill-defined with a short period between baseline and follow-up measures, and authors reported descriptive data rather than detailed or statistical analysis. Many papers had considerable heterogeneity of participants in regard to type and level of communication need.

\section{Intervention Outcomes}

The outcomes reported encompassed a wide range of measures, with the most commonly used being a count of initiation or response attempts using an AAC device (23 studies). Nine used linguistic analysis (such as Quantitative Production Analysis), and eight evaluated the effectiveness or intelligibility of communicative attempts. Four papers included the use of standardised language test scores, and two evaluated comprehension. There was a single study using rating of behaviour, one considered ease of use, four provided some description of use and one study rated client satisfaction.

\section{Participant Characteristics}

Participants included people with acquired non-progressive and progressive neurological disorders, autism/ autistic spectrum disorder, and other developmental disorders. There was considerable heterogeneity of participants in some studies, with diversity in terms of age, diagnosis, or pattern of communication difficulties.

\section{Acquired Non-Progressive Disorders}

The most commonly reported adult client group was use of high-tech AAC in people with aphasia resulting from a variety of non-progressive causes (14 studies) with all but one using computer software interventions. The largest group concerned aphasia resulting from a cerebrovascular accident. The only study evaluating a VOCA intervention [13] investigated the use of TouchSpeak ${ }^{\mathrm{TM}}$ in 30 people with severe aphasia following cerebrovascular accidents. Following the intervention 13 participants had no functional usage of the device, 5 were dependent users, 5 were independent users and 7 were extensive users. Another paper [14] outlined use of the SentenceShaper To $\mathrm{Go}^{\mathrm{TM}}$ portable device and found benefits in terms of the number of correctly used words and in the fluency of retelling a message. Evaluations of the SentenceShaper ${ }^{\mathrm{TM}}$ program [15-17] describe gains in narrative production, however, with little carry-over to spontaneous use. Four further papers [18-21] found positive outcomes with people who have aphasia, including gains in formal language assessments and grammatical structures. A single paper [22] described less beneficial outcomes from software interventions, reporting varying success in use of the CSpeak $^{\mathrm{TM}}$ Aphasia program. 
Four studies evaluated use of voice recognition computer programs in this client group. Two $[23,24]$ reported successful use of the DragonNaturallySpeaking ${ }^{\mathrm{TM}}$ program with a recognition accuracy of up to $65 \%$. Wade et al. [25] reported insufficient accuracy levels for reliable usage, although Dahl et al. [26] reported that problems of accuracy could be resolved by using SentenceShaper alongside voice recognition programs.

The use of AAC in people who have locked-in syndrome was described in four papers. One [27] outlined that 3 of the 6 participants continued to use the technology following completion of the trial. Papers by Lancioni et al. [28-30] evaluated use of a microswitch and computer with voice output device. The results indicated that frequency of responding was increased by using the system. One further paper relating to adults with non-progressive disorders [31] described the use of a laptop computer with word processing software for a male following total glossectomy and laryngectomy. The paper provided only general description regarding outcomes.

\section{Acquired Progressive Disorders}

Papers included in this review described interventions for people with amyotrophic lateral sclerosis/motor neurone disease, primary progressive aphasia and Alzheimer's disease.

Eight studies investigated use of brain-computer interface technologies for people with amyotrophic lateral sclerosis and found gains using the Thought Translation Device $^{\mathrm{TM}}$, an EEG-based (slow cortical shift) tool, enabling some participants to select letters to spell words [32-36], turn their communication device on and off [37, 38], or enable yes/no responses [39].

Pattee et al. [40] compared a text-to-speech AAC device to sign language for a person with primary progressive aphasia and apraxia of speech. They reported that there was a greater increase in correctly used words using sign language, and the participant rated the device as less useful and less easy to use than sign language. One paper [41] assessed usage in people with dementia, comparing a message board with pre-recorded speech output with no speech output. The results indicated that there were more single-word utterances and fewer total utterances when the AAC device included speech output.

\section{Autism/Autistic Spectrum Disorder}

Thirteen papers related to high-tech AAC use with people who have autism or autistic spectrum disorder. Studies by Olive et al. $[42,43]$ described positive effects such as increased total requesting and decreased episodes of challenging behaviour following milieu teaching combined with a VOCA, and Functional Communication Training and a VOCA. Schlosser et al. [44] investigated use of a VOCA for 5 children with autism requesting food at snack time, and found variable outcome across participants. Other studies [45-47] outlined evidence of positive impacts on requesting using a single recorded message on an SGD.

The effectiveness of different AAC interventions was compared in three studies. One [48] examined use of a SGD versus a Picture Exchange Communication System (PECS). Requesting increased using both systems, with preference based only on whichever was nearer. Other studies in this client group compared a SGD versus PECS and found little difference in outcomes between them [49], and compared peer-mediated naturalistic interventions with and without a SGD, reporting a significant increase in communicative behaviours using the SGD [50]. An additional paper [51] outlined that improvement in comprehension could result from use of a VOCA. The use of AAC by children with autism in the home setting was explored in three papers [52-54]. The authors of this work concluded that A AC could be used successfully and have beneficial effects on communication effectiveness and engagement rating.

\section{Cerebral Palsy}

Twelve papers were identified which described the use of AAC with children or adults who have cerebral palsy. One [55] which evaluated the introduction of Swedish DragonDictate $^{\mathrm{TM}}$ and Infovox ${ }^{\mathrm{TM}}$ systems outlined gains in recognition accuracy of $26-60 \%$. Another [56] assessing the use of DragonDictate reported that while 1 participant withdrew from the study, the other achieved gains in computer access efficiency of $40 \%$.

Hawley et al. [57] developed a limited vocabulary system with computerised training package for a home environment which achieved a recognition accuracy of around $95 \%$, operating around twice as fast as a switch control system. Another study [58] employed user movements to access a computer via a 'camera mouse' with 6 of the 10 participants able to use the technology to spell out communications. A system which detects minute facial muscle or eye movements in addition to brain waves, enabling movement of a computer cursor to make communication choices (Cyberlink ${ }^{\mathrm{TM}}$ ), was evaluated in one paper [59]. Two children achieved an $80 \%$ success rate in changing a picture on a computer using the system. An EEG-based brain-computer interface system was used by an adult with severe cerebral palsy with a $70 \%$ correct re- 
sponse rate for copy spelling following training in one study [601].

A Swedish study [61] described the use of Blissymbols ${ }^{\mathrm{TM}}$ on a SGD. The authors described progress with reading, writing and communication for the 2 child participants, however, outcomes were not clearly reported. Another study [62] described the use of TALK ${ }^{\mathrm{TM}}$, a text-storage and retrieval system. The single participant tested with the system achieved a conversational rate of 64 words per minute, and in another paper [63] the same authors described successful use of a pre-storage device. The Speech Enhanc$\mathrm{er}^{\mathrm{TM}}$ (a portable voice processor unit with speakers and feedback to the user) was found to be effective in improving intelligibility as rated by an experienced listener [64]. Other positive outcomes reported were increased unprompted use of a VOCA [65], and an increase in assertiveness, longer utterances, language and literacy [66].

\section{Other Developmental Disorders}

AAC interventions for children described as having multiple or complex developmental disorders were outlined in eleven papers. In one [67] gains in syntactic complexity using a SGD were outlined; however, pre-post test score change was smaller for the SGD than for a communication board. Interventions evaluated by Lancioni et al. [68-71] increased the number of utterances used by children with 'severe intellectual disability'.

DiCarlo and Banajee [72] assessed use of a VOCA with 2 adults, one diagnosed with Angelman syndrome and the other a chromosomal abnormality. The frequency of initiations during snack time increased for both participants (16-41 and 4-27\%) with a reduction in unclear initiations and prompted behaviours. Another paper [73] compared the effectiveness of PECS versus a VOCA. All participants increased the number of spontaneous requests during the intervention, half acquired use of PECs earlier and for half there was no difference between acquiring use of the VOCA versus PECS. Sevcik et al. [74] also reported positive outcomes following the introduction of a SGD, but with only a general description. Other studies described beneficial effects in a child with apraxia of speech [75]; a 3-year-old with lobar holoprosencephaly [76] and a 17-year-old with multiple disabilities [77].

\section{Discussion}

We reviewed the literature on interventions using high-tech AAC aids and identified 65 papers published in the last 10 years. Papers were found evaluating the use of a wide range of technology (SGDs/VOCA, voice output software on personal computers or laptops used as a communication aid, and technology which provides access to personal computers or laptops).

The findings of this review suggest that these devices may be beneficial to enhance communication across a broad range of diagnoses and age ranges. The reported variability in outcome within groups of individuals using the same system is important to note, however. There was a considerable range of outcome measures used by authors. Studies described devices enabling communication at very different levels, ranging from a yes/no response to sentence generation, with a predominance of frequency of utterance or accuracy measures. Some authors of included papers highlighted the limitations of baseline and follow-up measures and measures taken in controlled environments which did not take functional usage into account.

While we were able to identify a substantial body of papers evaluating interventions using high-technology AAC, there is currently a lack of high-quality evidence of effect. This is due to a lack of good-quality studies rather than there being evidence of a lack of effect. It is important to note the predominance of case series or case study designs in the field representing only level IV evidence [78]. There is currently a dearth of studies with comparator arms, which while presenting challenges must be a future priority if the evidence base is to be strengthened. While considered to be the design most subject to bias, case studies are commonly used and reported in the healthcare literature. It has been argued [12] that they can be a helpful source of information about adverse events, can generate hypotheses, provide more participants, longer follow-up and are more generalisable than controlled trials. However, they have significant limitations in terms of providing conclusive evidence of effectiveness. The evidence from these case studies should be used to underpin stronger designs in future research.

The review included a comprehensive search of electronic databases, and citation checking, but did not encompass hand searching of journals, which may have identified additional references. The definition of high technology versus low technology is subject to some lack of clarity in the literature and it is possible that papers of relevance were excluded using our criteria. The area where we considered that there was most potential for debate was in relation to the use of computer-based technology with people who have aphasia. We endeavoured to distinguish between software intended for intervention purposes only versus that designed to enable func- 
tional communication. Some of the programs identified (such as SentenceShaper) could be considered to be on the margins of AAC.

Much of the work, while describing generally positive outcomes (which may be attributable to publication bias), reports variability in response to intervention amongst participants. Future work would benefit from endeavouring to recruit using closer matching of individual communication levels rather than the tendency towards sampling by diagnostic category. There is an urgent requirement to extend our understanding of the needs and characteristics of clients who may or may not benefit from high-tech AAC technology. Some papers suggested that aspects such as visual semantic processing or cognitive functioning may impact the response to intervention. In particular, consideration needs to be given to controlling for attention as many of the studies cited above provided fairly intensive teaching and support of communication in general, which may have affected the outcomes with or without AAC. If characteristics of people who successfully use high-tech AAC can be identified, this would provide important information for decision-making regarding provision. Further research would also be helpful to compare the use of different devices, and the benefits of low-tech versus high-tech systems. There was the suggestion that for some individuals low-tech interventions may be more beneficial.

Many studies described their limitations in terms of being undertaken in a highly controlled context with lack of consideration of functional use and environmental factors. Issues relating to fidelity of the intervention re- gime may adversely impact outcomes outside these research environments. A minority of the papers evaluated use in a home context. Further work is needed exploring how effective the technology is in aiding communication in a functional setting.

Consideration of controlled versus more functional settings raises issues regarding the measurement of AAC intervention effectiveness. There is currently debate regarding what a successful outcome should be [79]. The identification of appropriate outcomes following intervention presents a significant challenge for the field. Outcomes could encompass successful introduction of a device, evaluation of client experiences, training of conversational partners, and/or ongoing satisfaction. The literature examined in this review used a wide variety of measures; however, many were frequency counts of communication utterances. It is recognised across the field of communication impairment that outcome measures need to cover a range of communication tasks and purposes, and that the individual's own views are important. A greater emphasis on developing functional measures of high-tech AAC intervention effectiveness seems warranted.

\section{Acknowledgements}

This study forms part of a research project commissioned and led by Communication Matters, the UK charity for augmentative and alternative communication. This work is funded by the $\mathrm{Na}-$ tional Lottery through Big Lottery Fund.

\section{References}

1 American Speech-Language-Hearing Association: Augmentative and Alternative Communication: Knowledge and Skills for Service Delivery, 2002. Available from www. asha.org/policy.

2 Campbell L, Balandin S, Togher L: Augmentative and alternative communication use by people with traumatic brain injury: a review. Adv Speech-Lang PA 2002;4:89-94.

-3 Schlosser R: Roles of speech output in augmentative and alternative communication: narrative review. Augment Altern Commun 2003;19:5-27.

4 McNaughton D, Bryen D: AAC technologies to enhance participation and access to meaningful societal roles for adolescents and adults with developmental disabilities who require AAC. Augment Altern Commun 2007;23:217-229.
5 Iacono $\mathrm{T}$, Lyon $\mathrm{K}$, West $\mathrm{D}$ : Non-electronic communication aids for people with complex communication needs. Int J Speech Lang Pathol 2011;13:399-410.

-6 Schlosser R, Wendt O: Effects of augmentative and alternative communication intervention on speech production in children with autism: a systematic review. Am J Speech Lang Pathol 2008;17:212-230.

7 Light J: Towards a definition of communicative competence for individuals using augmentative and alternative communication systems. Augment Altern Commun 1989;5: $137-144$

8 Schlosser R, Raghavendra P: Evidence-based practice in augmentative and alternative communication. Augment Altern Commun 2004;20:1-21.
9 Grant M, Booth A: A typology of reviews: an analysis of 14 review types and associated methodologies. Health Info Libr J 2009;26: 91-108.

10 Baxter S, Enderby P, Evans P, Judge S: Barriers and facilitators to use of high technology augmentative and alternative communication devices: a systematic review and qualitative synthesis. Int J Lang Commun Disord 2012;47:115-129.

11 West S, King V, Carey T, Lohr K, McKoy N, Sutton S, Lux L: Systems to Rate the Strength of Scientific Evidence. AHRQ Publication No 02-E016. Rockville, Agency for Healthcare Research and Quality, 2002. ,

12 Centre for Reviews and Dissemination: Systematic Reviews: CRD's Guidance for Undertaking Systematic Reviews in Health Care. York, University of York, 2007. 
13 Van de Sandt-Koenderman W, Wiegers J, Wielaert S, Duivenvoorden H, Ribbers G: High-tech AAC and severe aphasia: candidacy for TouchSpeak (TS). Aphasiology 2007;21:459-474.

14 Linebarger M, Romania J, Fink R, Bartlett M, Schwartz M: Building on residual speech: a portable processing prosthesis for aphasia. J Rehabil Res Dev 2008;45:1401-1414.

15 Linebarger M, Schwartz M: AAC for hypothesis testing and treatment of aphasic language production: lessons from a 'processing prosthesis'. Aphasiology 2005;10:930-942.

- 16 Fink R, Bartlett M, Lowery J, Linebarger M, Schwartz M: Aphasic speech with and without SentenceShaper: two methods for assessing informativeness. Aphasiology 2008;22: 679-690.

17 Albright E, Purves B: Exploring SENTENCESHAPER: treatment and augmentative possibilities. Aphasiology 2008;22:741752.

18 Hough M, Johnson R: Use of ACC to enhance linguistic communication skills in an adult with chronic severe aphasia. Aphasiology 2009;23:965-976.

-19 Johnson R, Hough M, King K, Vos P, Jeffs T: Functional communication in individuals with chronic severe aphasia using augmentative communication. Augment Altern Commun 2008;24:269-280.

20 Linebarger M, Schwartz M, Romania J, Kohn S, Stephens D: Grammatical encoding in aphasia: evidence from a processing prosthesis. Brain Lang 2000;75:416-427.

-21 Koul R, Corwin M, Hayes S: Production of graphic symbol sentences by individuals with aphasia: efficacy of a computer-based augmentative and alternative communication intervention. Brain Lang 2005;92:58-77.

-22 Nicholas M, Sinotte M, Helm-Estabrooks N: Using a computer to communicate: effect of executive function impairments in people with severe aphasia. Aphasiology 2005;19: 1052-1065.

23 Bruce C, Edmundson A, Coleman M: Writing with voice: an investigation of the use of a voice recognition system as a writing aid for a man with aphasia. Int J Lang Commun Disord 2003;38:131-148.

-24 Hux K, Rankin-Erickson J, Manasse N, Lauritzen E: Accuracy of three speech recognition systems: case study of dysarthric speech. Augment Altern Commun 2000;12000:186196.

-25 Wade J, Petheram B, Cain R: Voice recognition and aphasia: can computers understand aphasic speech? Disabil Rehabil 2001;23: 604-613.

26 Dahl D, Linebarger M, Berndt R: Improving automatic speech recognition of aphasic speech through the use of a processing prosthesis. Technol Disabil 2008;20:283-294.
7 Fager S, Beukelman D, Karantounis R, Jakobs T: Use of safe-laser access technology to increase head movement in persons with severe motor impairment: a series of case reports. Augment Altern Commun 2006;22: 222-229.

28 Lancioni G, Singh N, O'Reilly M, Sigafoos J, Buonocunto F, Sacco V et al: Microswitchand VOCA-Assisted Programs for Two PostComa Persons with Minimally Conscious State and Pervasive Motor Disabilities. Res Dev Disabil 2009;30:1459-1467.

29 Lancioni G, Singh N, O’Reilly M, Sigafoos J, Buonocunto F, Sacco V, et al: Two persons with severe post-coma motor impairment and minimally conscious state use assistive technology to access stimulus events and social contact. Disabil Rehab: Assist Technol 2009;4:367-372.

30 Lancioni G, Singh N, O’Reilly M, Sigafoos J, Buonocunto F, Sacco V, et al: Post-coma persons with motor and communication/consciousness impairments choose among environmental stimuli and request stimulus repetitions via assistive technology. Res Dev Disabil 2010;31:777-783.

31 Fox L, Rau M: Augmentative and alternative communication for adults following glosssectomy and laryngectomy surgery. Augment Altern Commun 2001;17:161-166.

\$2 Birbaumer N, Kubler A, Ghanayim N, Hin terberger T, Perelmouter J, Kaiser J, et al: The thought translation device (TTD) for completely paralyzed patients. IEEE Trans Rehabil Eng 2000;8:190-193.

-33 Kubler A, Furdea A, Halder S, Hammer E, Nijboer F, Kotchoubey B: A brain-computer interface controlled auditory event-related potential (p300) spelling system for lockedin patients. Ann NY Acad Sci 2009;1157:90100.

34 Kubler A, Neumann N, Kaiser J, Kotchoubey B, Hinterberger T, Birbaumer N: Brain-computer communication: self-regulation of slow cortical potentials for verbal communication. Arch Phys Med Rehabil 2001;82: 1533-1539.

35 Nijboer F, Sellers E, Mellinger J, Jordan M, Matuz T, Furdea A, Halder S, Mochty U, Krusienski D, Vaughan T, Wolpaw J, Birbaumer N, Kubler A: A P300-based brain-computer interface for people with amyotrophic lateral sclerosis. Clin Neurophysiol 2008; 119:109-1916.

-36 Sellers E, Kubler A, Donchin E: Brain-computer interface research at the University of South Florida cognitive psychophysiology laboratory: the P300 speller. IEEE Trans Neural Syst Rehabil Eng 2006;14:221-224.

37 Kaiser J, Kubler A, Hinterberger T, Neumann N, Birbaumer N: A non-invasive communication device for the paralyzed. Minim Invas Neurosurg 2002;45:19-23.
38 Kaiser J, Perelmouter J, Iversen I, Neumann N, Ghanayim N, Hinterberger T, et al: Selfinitiation of EEG-based communication in paralyzed patients. Clin Neurophysiol 2001; 112:551-554.

-39 Neshige R: Communication aid device utilizing event-related potentials for patients with severe motor impairment. Brain Res 2007;1141:218-227.

40 Pattee C, Von Berg S, Ghezzi P: Effects of alternative communication on the communicative effectiveness of an individual with a progressive language disorder. Int J Rehabil Res 2006;29:151-153.

41 Fried-Oken M, Rowland C, Baker G, Dixon M, Mills C, Schultz D, Oken B: The effect of voice output on AAC-supported conversations of persons with Alzheimer's disease. ACM T Access Comput 2009;1: article 15.

42 Olive M, de la Cruz B, Davis T, Chan J, Lang R, O'Reilly M, Dickson S: The effects of enhanced milieu teaching and a voice output communication aid on the requesting of three children with autism. J Autism Dev Disord 2007;37:1505-1513.

43 Olive M, Lang R, Davis T: An analysis of the effects of functional communication and a Voice Output Communication Aid for a child with autism spectrum disorder. Res Autism Spectrum Disord 2008;2:223-236.

44 Schlosser R, Sigafoos J, Luisell J, Angermeier K, Harasymowzy U, Schooley K, Belfiore P: Effects of synthetic speech output on requesting and natural speech production in children with autism: a preliminary study. Res Autism Spectrum Disord 2007;1:139163.

45 Sigafoos J, Drasgow E, Halle J, O’Reilly M, Seely-York S, Edrisinha C, Andrews A: Teaching VOCA use as a communicative repair strategy. J Autism Dev Disord 2004;34: 411-422.

46 Sigafoos J, O’Reilly M, Seely-York S, Edrisinha C: Teaching students with developmental disabilities to locate their AAC device. Res Dev Disabil 2004;25:371-383.

-47 Sigafoos J, Didden R, O'Reilly M: Effects of speech output on maintenance of requesting and frequency of vocalizations in three children with developmental disabilities. Augment Altern Commun 2003;19:37-47.

48 Sigafoos J, Green V, Payne D, Son S, O’Reilly M, Lancioni G: A comparison of picture exchange and speech-generating devices: acquisition, preference, and effects on social interaction. Augment Altern Commun 2009;25:99-109.

49 Son S, Sigafoos J, O'Reilly M, Lancioni G: Comparing two types of augmentative and alternative communication systems for children with autism. Pediatr Rehabil 2006;9: 389-395.

-50 Trembath D, Balandin S, Togher L, Stancliffe $\mathrm{R}$ : Peer-mediated teaching and augmentative and alternative communication for preschool-aged children with autism. J Intellect Dev Disabil 2009;34:173-186. 
51 Brady N: Improved comprehension of object names following voice output communication aid use: two case studies. Augment Altern Commun 2000;16:197-204.

52 Thunberg G, Ahlsen E, Dahlgren Sandberg A: Children with autistic spectrum disorders and speech-generating devices: communication in different activities at home. Clin Linguist Phon 2007;21:457-479.

53 Thunberg G, Dahlgren Sandberg A, Ahlsen E: Speech-generating devices used at home by children with autism spectrum disorders: a preliminary assessment. Focus Autism Other Dev Disabil 2009;24:109-114.

54 Sigafoos J, O’Reilly M, Seely-York S, Weru J, Son S, Green V, Lancioni G: Transferring AAC intervention to the home. Disabil Rehabil 2004;26:1330-1334.

55 Raghavendra P, Rosengren E, Hunnicutt S: An investigation of different degrees of dysarthric speech as input to speaker-adaptive and speaker-dependent recognition systems. Augment Altern Commun 2001;17:265-275.

-56 Havstam C, Buchholz M, Hartelius L: Speech recognition and dysarthria: a single subject study of two individuals with profound impairment of speech and motor control. Logoped Phoniatr Vocol 2003;28:81-90.

57 Hawley M, Enderby P, Green P, Cunningham S, Brownsell S, Carmichael J, et al: A speechcontrolled environmental control system for people with severe dysarthria. Med Eng Phys 2007;29:586-593.

58 Betke M, Gips J, Fleming P: The camera mouse: visual tracking of body features to provide computer access for people with severe disabilities. IEEE Trans Neurol Syst Rehabil Eng 2002;10:1-10.

59 Redstone F: A training program for the use of the Cyberlink control system for young children with cerebral palsy. Technol Disabil 2006;18:107-115.

-60 Neuper C, Muller G, Kubler A, Birbaumer N, Pfurtscheller G: Clinical application of an EEG-based brain-computer interface: a case study in a patient with severe motor impairment. Clin Neurophysiol 2003;11:399-409.
61 Ferm U, Amberntson B, Thunberg G: Development and evaluation of a MINSPEAK application using Blissymbols: experiences from two case studies. Augment Altern Commun 2001;17:233-244.

62 Todman J: Rate and quality of conversations using a text-storage AAC system: single-case training study. Augment Altern Commun 2000;16:164-179.

63 File P, Todman J: Evaluation of the coherence of computer-aided conversations. Augment Altern Commun 2002;18:228-241.

64 Bain C, Ferguson A, Mathisen B: Effectiveness of the Speech Enhancer on intelligibility: a case study. J Med Speech-Lang Pathol 2005;13:85-95.

65 Evans Cosbey J, Johnston S: Using a SingleSwitch Voice Output Communication Aid to Increase Social Access for Children with Severe Disabilities in Inclusive Classrooms. Res Pract Persons Severe Disabil 2006;31: 144-156.

66 Mathisen B, Arthur-Kelly M, Kidd J, Nissen C: Using MINSPEAK: a case study of a preschool child with complex communication needs. Disabil Rehab: Assist Technol 2009;4 376-383.

67 Bruno J, Trembath D: Use of aided language stimulation to improve syntactic performance during a weeklong intervention program. Augment Altern Commun 2006;22: 300-313.

-68 Lancioni G, O’Reilly M, Singh N, Sigafoos J, Oliva D, Severini L: Three persons with multiple disabilities accessing environmental stimuli and asking for social contact through microswitch and VOCA technology. J Intellect Disabil Res 2008;52:327-336.

69 Lancioni G, Singh N, O’Reilly M, Oliva D Montironi G, Piazza F, et al: Using computer systems as microswitches for vocal utterances of persons with multiple disabilities. Res Dev Disabil 2004;25:183-192
70 Lancioni G, Singh N, O’Reilly M, Sigafoos J, Oliva D, Montironi G: Evaluating a computer system used as a microswitch for word utterances of persons with multiple disabilities. Disabil Rehabil 2004;26:1286-1290.

71 Lancioni G, Singh N, O'Reilly M, Oliva D, Montironi G: A computer system serving as a microswitch for vocal utterances of persons with multiple disabilities: two case evaluations. Research report. J Vis Impair Blindness 2004;98:116-120.

72 DiCarlo C, Banajee M: Using voice output devices to increase initiations of young children with disabilities. J Early Interv 2000;23: 191-199.

73 Bock S, Stoner J, Beck A, Hanley L, Prochnow J: Increasing functional communication in non-speaking pre-school children: comparison of PECS and VOCA. Educat Train Dev Disabil 2005;40:264-278.

74 Sevcik R, Romski M, Adamson L: Research directions in augmentative and alternative communication for preschool children. Disabil Rehabil 2004;26:1323-1329.

75 Bornman J, Alant E, Meiring E: The use of a digital voice output device to facilitate language development in a child with developmental apraxia of speech: a case study. Disabil Rehabil 2001;23:623-634.

76 Romski M, Haarbauer-Krupa J, Cheslock M, Sevcik R, Adamson L: Language and communication changes in a child with holoprosencephaly: a case report. J Med SpeechLang Pathol 2008; 16:77-82.

77 Dyches T, Davis A, Lucido B, Young J: Generalization of skills using pictographic and voice output communication devices. Augment Altern Commun 2002;18:124-131.

78 Chan K, Bhandari M: Three minute critical appraisal of a case series article. Indian J Orthop 2011;45:103-104.

79 Lund S, Light J: Long-term outcomes for individuals who use augmentative and alternative communication. Part I. What is a 'good' outcome? Augment Altern Commun 2006; 22:284-299. 